\title{
Simulation numérique d'un écoulement stratifié turbulent
}

\author{
Victor ROSALES ${ }^{1,2}$, Antoine GARAPON ${ }^{1}$, Daniel LEVACHER ${ }^{2}$ \\ ${ }^{1} E D F$ R\&D - Laboratoire National d'Hydraulique et Environnement \\ 6, Quai Watier BP 4978401 Chatou, Cedex e-mail : \\ victor_rs@hotmail.com,tél.0130877266 \\ ${ }^{2}$ Université de Caen,M2C-GRGC,UMR 6143 CNRS, 24, rue des \\ Tilleuls, 14000 Caen.
}

\section{Résumé}

On présente dans cet article la modélisation numérique d'un écoulement turbulent dans un canal composé de deux couches ayant des vitesses et des températures différentes, définissant des cas de stratification stable ou instable. Cette étude a été réalisée expérimentalement par Viollet (1980). On se propose de vérifier et valider, par une approche systématique définie par Colleman et Stern (1997), le code de simulation TELEMAC 3D (Janin et al., 1992) grâce aux résultats de Viollet, et d'effectuer un analyse de l'influence de différents paramètres de calcul.

\begin{abstract}
This paper concerns a numerical simulation of a two layers stratified turbulent flow in a flume. Different temperatures and velocities have been chosen for the layers to obtain a stable or an unstable stratification. The experimental study has been conducted by Viollet (1980). Considering a systematic approach proposed by Colleman and Stern (1997), and using the experimental results of Viollet, a validation of TELEMAC 3D simulation code (Janin et al ; 1992) has been carried out. The influence of some numerical parameters is analyzed.
\end{abstract}

\section{Introduction}

Le code TELEMAC 3D (Janin et al., 1992), développé à EDF-LNHE est appliqué à la modélisation des écoulements à surface libre. Il résout les équations de Navier-Stokes Reynolds avec ou sans hypothèse hydrostatique ainsi que l'équation de transport-diffusion de traceur. L'algorithme de calcul repose sur la technique de pas de temps fractionnaires et la discrétisation spatiale est effectuée par la méthode des éléments finis. Différents modèles de turbulence peuvent être utilisés (longueur de mélange avec ou sans effet de flottabilité, $\mathrm{k}-\varepsilon$ ). 


\section{Dëfinitions}

La méthode de vérification et validation de Colleman et al, prend en compte les rangs des incertitudes dues aux erreurs provenant de la simulation numérique et des données expérimentales pour calculer les limites de validité des comparaisons.

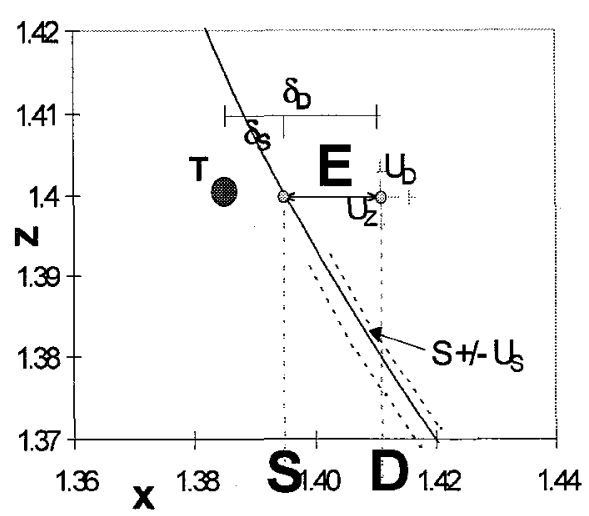

Figure 1 Définition de l'erreur de comparaison. Comparison error definition.

L'erreur $\delta$ est la différence entre un résultat de la simulation $S$ (Figure 1) ou expérimentale $D$ et la valeur réelle $T$. L'erreur de simulation $\delta_{S}$ est la différence entre $S$ et $T$, tandis que, $\delta_{D}$ est la différence entre $D$ et $T$. $E$ représente l'erreur accumulée de toutes les erreurs associées à la simulation et à l'expérimentation.

L'incertitude $U$ est l'estimation de l'erreur $E$ tel que le rang $\pm U$ contienne les $95 \%$ de la valeur réelle ; cet intervalle indique la amplitude, pas le signe de l'erreur. Dans certaines conditions, le signe de l'erreur $\delta$ peut se calculer;

l'incertitude global s'écrit alors:

$$
U_{V}^{2}=U_{D}^{2}+U_{S N}^{2}
$$

L'incertitude de validation $U_{V}$, est utilisée. Elle est estimée à partir des incertitudes qu' on peut calculer. Si la valeur absolue de $|E|<U_{V}$ la validation est accomplie. La valeur $U_{S N}$ est composée des incertitudes numériques (liées à la discrétisation, à la dissipation artificielle, à la perte de masse, etc), et de modélisation (géométrie approchée, résolution des équations, conditions limites, modèles de turbulence, etc); toutes les deux ayant une complexité importante. $U_{D}$ est l'incertitude des résultats expérimentaux (liée aux méthodes et équipement).

\section{Description du cas test}

A partir de l'expérience de Viollet, on a reproduit numériquement un canal ouvert à surface libre $(0,4 \mathrm{~m}$ de largeur et $11 \mathrm{~m}$ de longueur) avec deux couches d'eau (d'épaisseur $h=0.1 \mathrm{~m}$ ) de vitesses et températures différentes : stratification stable $\left(u_{1}=0,05 \mathrm{~m} / \mathrm{s}, u_{2}=0,1 \mathrm{~m} / \mathrm{s}, T_{1}=26,6^{\circ} \mathrm{C}\right.$ et $\left.T_{2}=12^{\circ} \mathrm{C}\right)$, stratification instable $\left(u_{1}\right.$ $=0,1 \mathrm{~m} / \mathrm{s}, u_{2}=0,05 \mathrm{~m} / \mathrm{s}, T_{1}=12^{\circ} \mathrm{C}$ et $T_{2}=26.6^{\circ} \mathrm{C}$ ). On dispose comme données expérimentales, des profils de vitesse et de température à $1 \mathrm{~m}$ et $4 \mathrm{~m}$ de l'entrée du canal, voir Figure 2. 


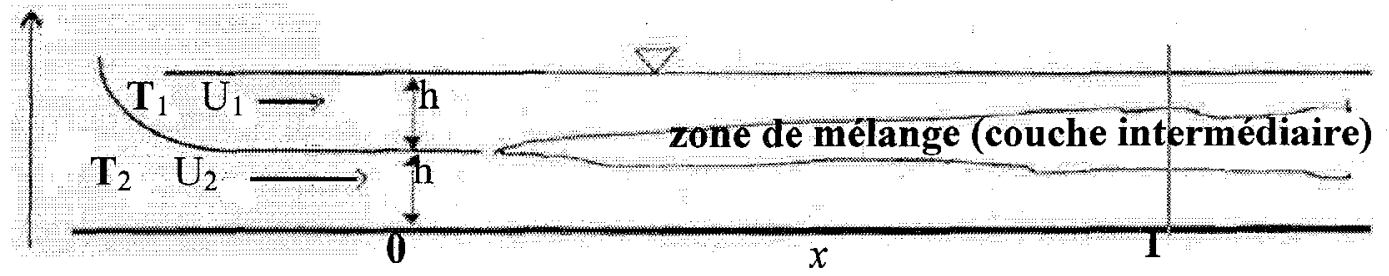

Figure 2 Schéma du modèle expérimental. Experimental scheme

L'intérêt de cette configuration expérimentale est d'utiliser le nombre de Froude réduit dont l'écart de températures est caractérisé par :

$$
F r=\frac{\left|u_{1}-u_{2}\right|}{\sqrt{g h \beta\left|T_{1}-T_{2}\right|}}
$$

où $g$ est la pesanteur de la gravité et $\beta$ égal à $2.10^{-4}$ est le coefficient de dilatation volumique. Dans le cas stable, lorsque le nombre de Froude réduit diminue, l'effet de densité augmente provoquant une diminution du mélange entre les deux couches. Alors les deux couches persistent et conservent leur température sans se mélanger sur une grande longueur. Cependant, les vitesses s'homogénéisent. Ceci est dû aux ondes qui se produisent à l'interface entre les écoulements générant un certain échange de quantité de mouvement même s'il n'y a pas d'échange de masse ou de quantité de chaleur. Par contre, dans le cas instable, le mélange turbulent augmente à cause de l'effet de densité, c'est alors le phénomène inverse.

\section{Modèle numérique}

Suivant la méthode de Colleman et al, 4 maillages de calcul ont été utilisés pour l'étape de vérification. Les maillages ont un rapport de taille et de pas de temps associé $r_{g}=\sqrt{2}$. La taille des maillages est organisée, de la plus grossière, notée $M 4$, à la plus fine, notée $M 1$, Le rapport $r_{g}$ a été fixé sur le pas de temps, l'axe $x$ et l'axe $z$; (sur l'axe $y$ il reste uniforme parce que il n'y a pas d'effets de diffusion latéraux, voir Tableau 1).

\begin{tabular}{|c|c|c|c|c|c|c|c|c|c|c|}
\hline & \multicolumn{3}{|c|}{ Nombre de points } & \multicolumn{3}{|c|}{$\begin{array}{c}\text { Taille de cellule } \\
(\mathrm{m})\end{array}$} & \multicolumn{3}{|c|}{$\begin{array}{c}\text { Taille par rapport à } \\
\text { l'épaisseur }\end{array}$} & $\begin{array}{l}\text { pas de } \\
\text { temps }(s)\end{array}$ \\
\hline Maillage & $x$ & $y$ & $z$ & $\Delta x$ & $\Delta y$ & $\Delta z$ & $\Delta x / h$ & $\Delta y / h$ & $\Delta z / h$ & $t$ \\
\hline M1 & 64 & 4 & 56 & 0.172 & 0.100 & 0.004 & 1.719 & 1.000 & 0.036 & 0.10 \\
\hline M2 & 44 & 4 & 40 & 0.250 & 0.100 & 0.005 & 2.500 & 1.000 & 0.050 & 0.14 \\
\hline M3 & 32 & 4 & 28 & 0.344 & 0.100 & 0.007 & 3.438 & 1.000 & 0.071 & 0.20 \\
\hline M4 & 22 & 4 & 20 & 0.500 & 0.100 & 0.010 & 5.000 & 1.000 & 0.100 & 0.28 \\
\hline
\end{tabular}

Tableau 1 Caractéristiques spatiales des 4 maillages. 4 grid spatial characteristics

On a sélectionné les modèles de longueur de mélange $(\mathrm{Lm})$ et $k-\varepsilon$ pour la turbulence verticale, la valeur du coefficient de frottement suivant la loi de 
Strickler a été de 45 . Le temps total de calcul a été fixé à $700 \mathrm{~s}$. Deux couches turbulentes sont présentes, d'une part, la couche de mélange au milieu des deux écoulements, et d'autre part, celle du fond. La stabilité et la convergence du modèle est vérifiée avec l'équilibre du profil de la surface libre, les isovaleurs de vitesses tridimensionnelles, la viscosité et le nombre de Richardson qui définit la stratification.

\section{Vérification (résultats de la vitesse pour le cas de stratification stable avec le modèle Lm)}

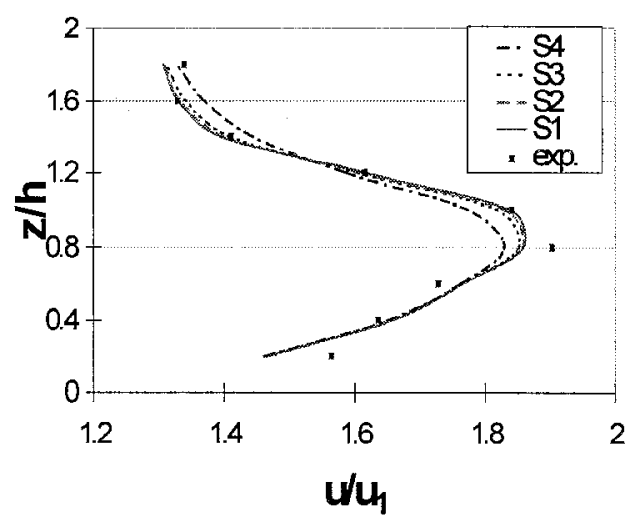

Figure 3 Profil de vitesses des 4 maillages à la station $x=4 \mathrm{~m} .4$ grid velocity profile at $x=4 \mathrm{~m}$
Les valeurs de vitesse à la station $x=4 m$ (Figure 3) sont extraites pour appliquer la méthode de vérification. Chaque ligne représente la solution obtenue après convergence à $700 \mathrm{~s}$ de calcul pour chaque maillage, l'axe $x$ représente la valeur de la vitesse adimensionnée par rapport à la vitesse $u_{1}$ et l'axe $y$ représente la valeur adimensionnelle de la profondeur $z$ par rapport à l'épaisseur de la couche $h$.

Les profils de vitesses convergent vers la solution $S 1$ résultant du maillage $M 1$. On peut voir aussi un point d'inflexion à la hauteur $z / h=$ 1.28 qui limite la couche de mélange de la couche supérieure.

L'étape de vérification comprend l'évaluation de l'incertitude itérative $U_{l}$, variation de la solution d'un même maillage une fois atteint la convergence en temps, et l'incertitude de maillage $U_{g}$, variation de la solution entre deux maillages lorsqu'ils convergent. L'incertitude de simulation numérique $U_{s n}$ est la somme de l'incertitude itérative et de maillage.

On commence par calculer l'incertitude itérative ; c'est a dire la variation pour un calcul de la solution aux dernières itérations :

$$
U_{I}=1 / 2\left(S_{U}-S_{L}\right)
$$

$U_{I}$ est l'incertitude itérative, $S_{U}$ et $S_{L}$ sont les valeurs supérieure et inférieure de la variation itérative. A la Figure 4, on voit les différentes incertitudes itératives obtenues sur la vitesse en utilisant chaque maillage. La solution du maillage $3, M 3$, a une incertitude itérative plus faible, tandis que $M 1$ a le plus d'incertitude. 


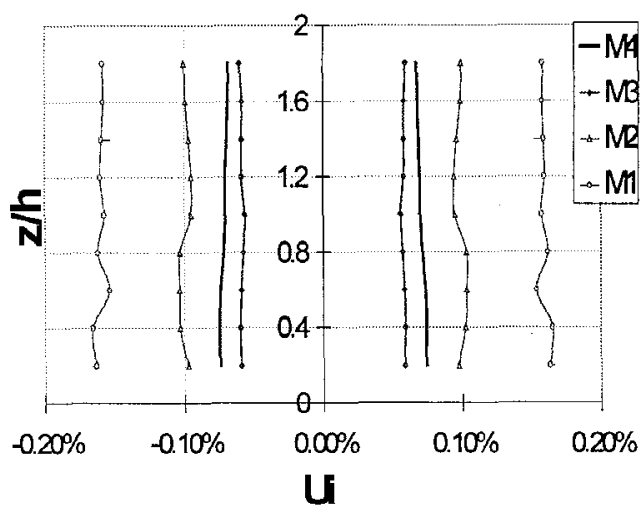

Figure 4 Incertitude itérative. intéressés à une valeur globale de l'erreur. Iterative uncertainty

maillage.

$$
\left|\varepsilon_{21}\right|_{R M S}=\sqrt{\frac{\left[\sum_{i=1}^{N}\left(S_{i 2}-S_{i 1}\right)^{2}\right]}{N}}
$$

Le indice $i$ dénote différents valeurs sur la vertical pour $N$ points de maillage. Les valeurs trouvées se rangent ainsi : $\left|\varepsilon_{43}\right|_{R M S}>\left|\varepsilon_{32}\right|_{R M S}>\left|\varepsilon_{21}\right|_{R M S}$ ce qui atteste du raffinement du maillage. Puis le rayon de convergence $R_{g}=\varepsilon_{21} / \varepsilon_{32}$ indiquant le type de convergence atteint par l'étude, est calculé. Il y a trois conditions de convergence possibles pour un étude de trois maillages.

(i) Convergence monotone : $0<R_{k}<1$

(ii) Convergence Oscillatoire : $R_{k}<0$

(iii) Divergence : $R_{k}>1$

Il faut dire que le fait de utiliser une valeur globale de l'erreur limite la condition de convergence oscillatoire parce que la valeur de $R_{k}$ sera toujours supérieur à 1 . On a donc vérifié d'abord que cette condition n'était pas prédominante en analysant certaines points du maillage. Dans notre cas, on a $R_{g 31}=\left|\varepsilon_{32}\right|_{R M S}\left|\varepsilon_{21}\right|_{R M S}=0.697$ (rayon de convergence entrez les mailles 3 et 1 ) et $R_{g 42}=\left|\varepsilon_{43}\right|_{R M S}\left|\varepsilon_{32}\right|_{R M S}=0.268$ (rayon de convergence entrez les mailles 4 et 2 ). La condition $R_{g}<1$, donne une convergence strictement monotone. De plus, il faut noter que l'inverse du rapport de taille $r_{g}$ est égal à $1 / \sqrt{2}$ soit 0.707 . Ceci est très proche du rayon de convergence $R_{g 31}$ (étude 3-1). Ensuite, on utilise la méthode relative au cas de convergence monotone à laquelle on applique l'extrapolation générale de Richardson pour estimer l'incertitude $U_{g}$, l'erreur estimée $\delta_{g}^{*}$ et l'incertitude de cette erreur $U_{g c}$ par rapport aux trois maillages. En considérant les 
3 maillages, on peut calculer l'ordre de précision $P_{g}$ et le facteur de correction $C_{g}$.

Le calcul pour le maillage $M 1$ est détaille, comme suit :

$$
P_{g}=\frac{\ln \left(\varepsilon_{32} / \varepsilon_{21}\right)}{\ln \left(r_{g}\right)}=1.041 \quad C_{g}=\frac{r_{g}^{P_{g}}-1}{r_{g e s t}^{P_{g e s t}}-1}=1.048
$$

avec $P_{g}$ estimé noté $P_{g e s t}$ égal à 1 et $r_{g}$ estimé noté $r_{g e s t}$ égal à $\gamma 2$. On évalue l'erreur de maillage $\delta_{\mathrm{g} 1}^{*}$ pour $M 1$ au moyen de l'extrapolation générale de Richardson $\delta_{R E g l}^{*}$, à savoir :

$$
\delta_{R E_{g 1}}^{*}=\frac{\varepsilon_{21}}{r_{g}^{P_{g}}-1} \quad \delta_{g 1}^{*}=C_{g} \delta_{R E_{g 1}}^{*}
$$

Puis, l'incertitude de maillage $U_{g}$ et l'incertitude de l'erreur estimée $U_{g c}$

$$
\mathrm{U}_{\mathrm{g}}=\mathrm{C}_{\mathrm{g}} \delta_{R E_{g 1}}^{*}+\left|\left(1-\mathrm{C}_{\mathrm{g}}\right) \delta_{R E_{g 1}}^{*}\right| \quad \mathrm{U}_{\mathrm{gc}}=\left|\left(1-\mathrm{C}_{\mathrm{g}}\right) \delta_{R E_{g 1}}^{*}\right|
$$

La Figure 5 illustre les différentes incertitudes de calcul. L'incertitude itérative est plus petite que celle de maillage qui est beaucoup plus réduite quand on applique la correction. D'ailleurs, les points les plus incertains sont ceux qui se trouvent dans la couche de mélange, entre les deux écoulements.

\begin{tabular}{|c|c|c|c|c|c|c|c|c|}
\hline Etude & $\mathrm{R}_{\mathrm{g}}$ & $\mathrm{E}_{\mathrm{g}}$ & $\mathrm{P}_{\mathrm{g}}$ & $\mathrm{C}_{\mathrm{g}}$ & $\delta_{\mathrm{g} 1}$ & $\mathrm{U}_{\mathrm{i}}$ & $\mathrm{U}_{\mathrm{g}}$ & $\mathrm{U}_{\mathrm{gc}}$ \\
\hline $\mathrm{M} 1-3$ & 0.6972 & $0.45 \%$ & 1.0408 & 1.0487 & $1.08 \%$ & $0.10 \%$ & $0.71 \%$ & $0.03 \%$ \\
\hline $\mathrm{M} 2-4$ & 0.2682 & $0.64 \%$ & 3.7971 & 6.5869 & $1.55 \%$ & $0.06 \%$ & $1.84 \%$ & $0.84 \%$ \\
\hline
\end{tabular}

Tableau 2 Tableau de vérification de la vitesse adimensionnelle $\left(u / u_{1}\right)$. Adimensional velocity verification $\left(u / u_{1}\right)$

On présente les paramètres de validation dans le tableau 2. La vérification est accomplie dans les deux études. Le rayon de convergence a une valeur inférieure à 1 , ce qui représente bien une convergence globale monotone.
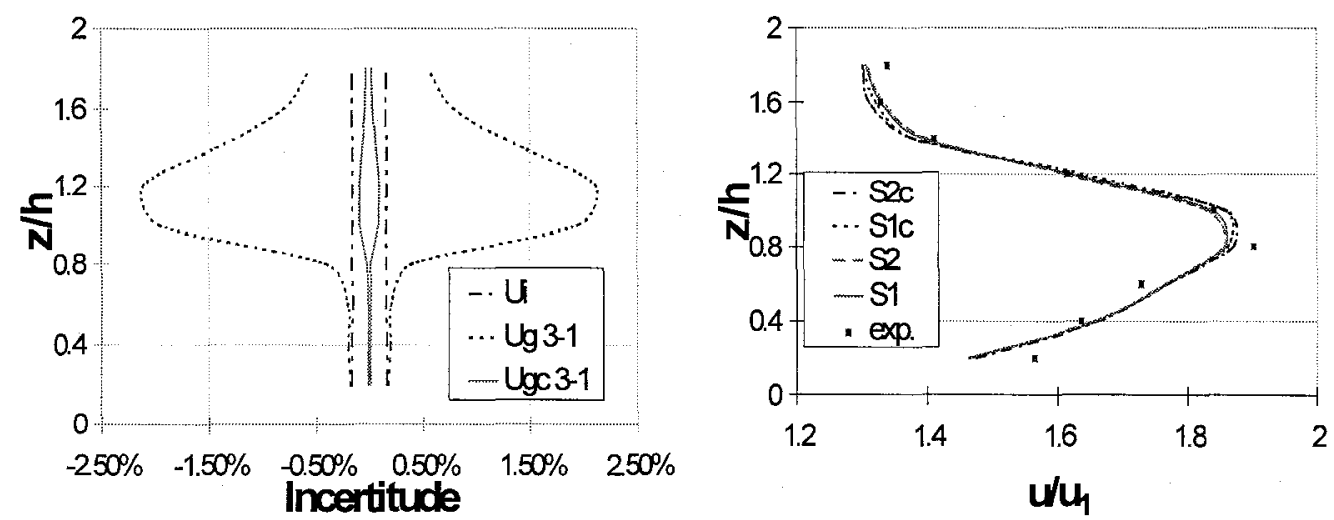

Figure 5 Incertitudes de simulation Figure 6 Solutions corrigés des numérique. Numerical simulation profils de vitesse. Corrected solutions uncertainties

for velocity profiles 
Les erreurs et incertitudes sont plus petites dans l'étude 3-1 que dans l'étude 4-2 montrant ainsi l'efficacité du raffinement sauf pour l'incertitude itérative dont l'utilisation de maillages plus raffinés peut augmenter cette valeur. On peut constater dans l'étude 3-1 que l'ordre de précision $P_{g}$ et le facteur de correction $C_{g}$ sont proches des valeurs asymptotiques $P_{\text {gest }}=1 ; C_{g}=1$. Augmenter le raffinement ne servirait à rien.

Les résultats indiquent que l'étude des maillages 3-1 a des valeurs d'incertitudes et erreurs plus petites par rapport a l'étude des maillages 4-2. Connaissant l'amplitude et le signe de l'erreur de la simulation numérique $\delta_{g l}$, on la soustrait obtenant alors la solution corrigée : $S_{C}=S-\delta_{g 1}$, comme le montre la Figure 6 pour les solutions $S 1$ et $S 2$.

\section{Validation (résultats de la vitesse pour le cas de stratification stable avec le modèle Lm)}

L'étape de validation permet de savoir si les résultats des simulations se trouvent dans les limites de l'incertitude de validation $U_{V}$ qui prend en compte l'incertitude numérique $U_{s n}$ et expérimentale $U_{D}$. Rappelons que l'incertitude numérique totale $U_{s n}$ est la somme des incertitudes d'itération $U_{I}$ et de maillage $U_{g}$.

On estime l'incertitude expérimentale sachant que les données ont été prises avec un micro-moulinet étalonné avec l'équation : $y=1.6 x-4$ où $y$ est le nombre de passages de pale pendant une période de $100 \mathrm{~s}$ et $x$ est la vitesse en $\mathrm{m} / \mathrm{s}$. La différence de vitesse entre deux passages de pale est $1 / 1.6=0.625 \mathrm{~m} / \mathrm{s}$, correspondant à une incertitude $U_{D}=12.5 \%$ pour $u_{1}=0.05 \mathrm{~m} / \mathrm{s}$. L'incertitude expérimentale est beaucoup plus importante que l'incertitude numérique à cause de la précision de la mesure du micro-moulinet.

On détermine alors 1'incertitude de validation $U_{V}^{2}=U_{s n}^{2}+U_{D}^{2}$ pour chaque point du profil et aussi l'incertitude de validation pour la solution corrigé $U_{V c}$.

Finalement, on observe le comportement des erreurs $E=D-S$ par rapport aux limites de validation $U_{V}$ (Figure 7). Tous les points sont validés car ils sont situés à l'intérieur des limites. Les erreurs corrigées sont également

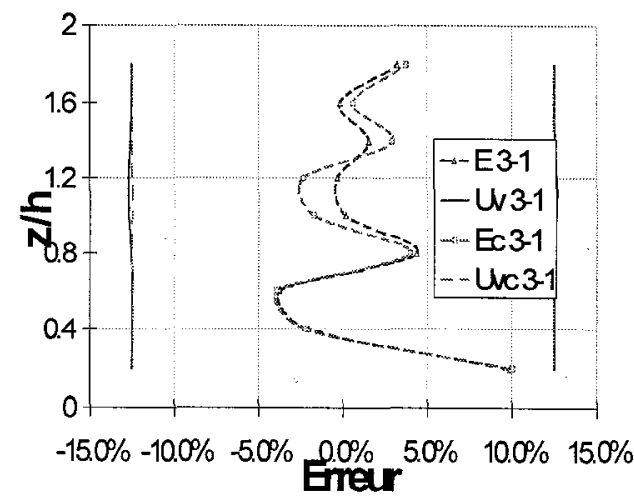

Figure 7 Validation de la convergence pour la vitesse étude 31. Velocity convergence validation for study 3-1 
validées $E c=D-S_{C}$. Le point pour lequel l'erreur est plus grande, est celui situé prés du fond. Ceci est probablement causé par la formulation de la fonction de la paroi dans le modèle de turbulence où la couche turbulente est sensiblement modifiée.

\section{Vérification et validation des autres résultats}

En ce qui concerne les résultats de température, la convergence est observée pour les deux études, le rayon de convergence $R_{g}$ est inférieur à 1 , l'erreur de maillage $E_{g}$ diminue en le raffinant de la même façon que l'erreur estimé $\delta$. On a dissocié du calcul le point $z / h=l$ (Figure 8), parce qu'il s'agit d'un point d'inflexion où l'erreur est indéfinie.

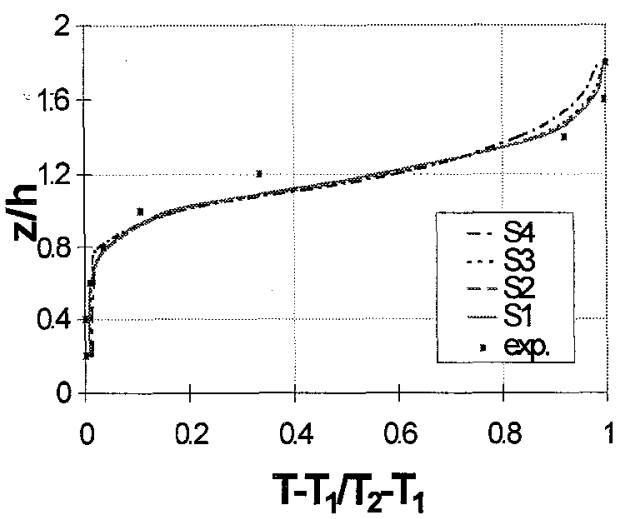

Figure 8 Profils de température $x=4 \mathrm{~m}$ pour le cas de stratification stable avec le modèle $\mathrm{Lm}$. Temperature profiles at $x=4 m$ for stable stratification using Lm model

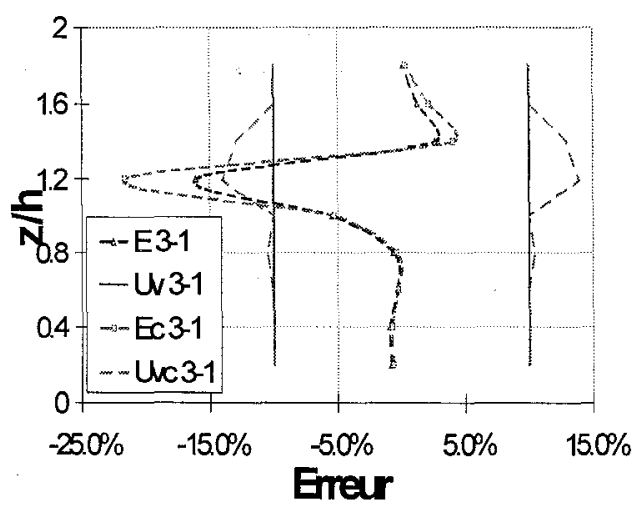

Figure 9 Validation de la température pour le cas de stratification stable avec le modèle Lm. Temperature convergence for stable stratification using Lm model

$\mathrm{Vu}$ que l'erreur expérimentale a été estimé à 10\%, Salaün (1980), la validation a été accomplie sauf pour le point $\mathrm{z} / \mathrm{h}=1.2$, Figure 9 , situé dans la zone de mélange. Ça nous indique que la solution dans la zone de mélange n'est pas encore optimisé.

Pour les simulations de la stratification instable, les effets des forces de gravité provoquent un mélange total des couches, on peut voir à la figure 10 les profils de vitesse. Bien qu'ils soient différents des valeurs expérimentales au niveau de la surface libre, l'erreur se trouve toujours dans les limites de validation, voir Figure 11. La Figure 12 montre le mélange de la température ce qui corresponde aux mesures en laboratoire. Une validation correcte est observée pour tous les points du profil, voir Figure 13. 


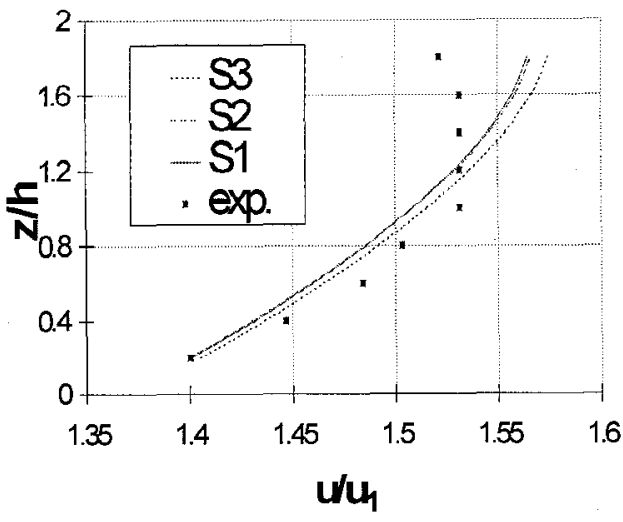

Figure 10 Profils de vitesse $x=4 \mathrm{~m}$ pour le cas de stratification instable avec le modèle $\mathbf{L m}$. Velocity profiles at $x=4 m$ for unstable stratification using Lm model

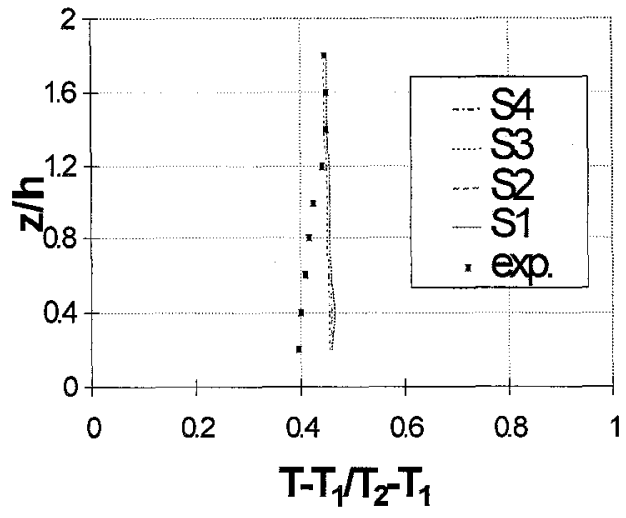

Figure 12 Profils de température $x=4 \mathrm{~m}$ pour le cas de stratification instable avec le modèle $\mathbf{L m}$. Temperature profiles at $x=4 m$

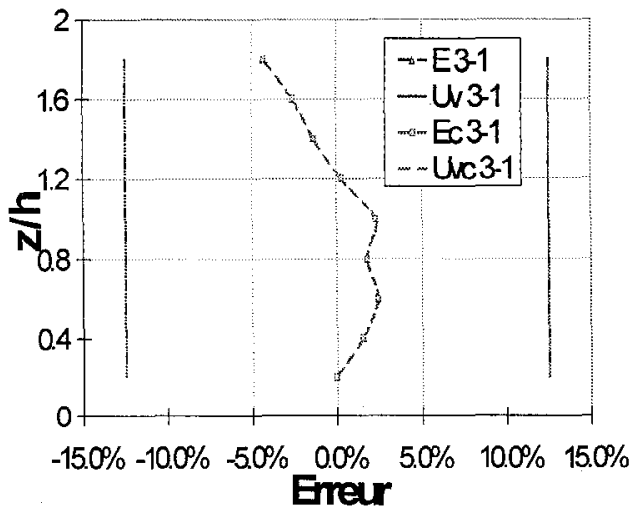

Figure 11 Validation de la vitesse pour le cas de stratification instable avec le modèle $\mathrm{Lm}$. Velocity convergence for unstable stratification using Lm model

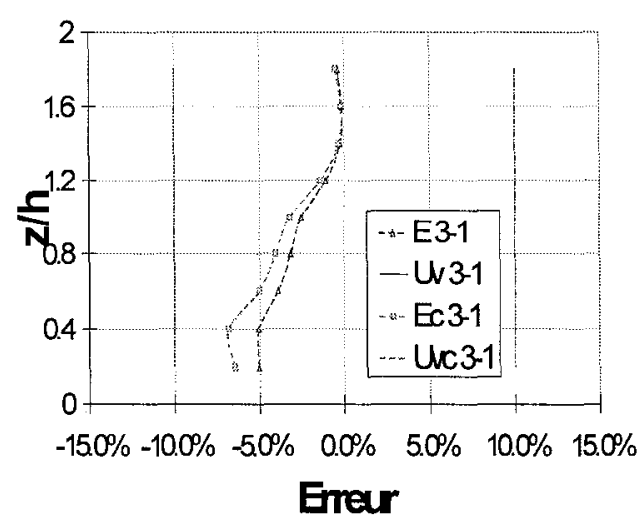

Figure 13 Validation de la température pour le cas de stratification instable avec le modèle Lm. Velocity convergence validation for study 3-I

Le modèle k- $\varepsilon$ a été testé pour le cas de stratification stable. La Figure 14 montre les profils de vitesse résultants. On remarque une surestimation à la zone de mélange de la même manière que le modèle à longueur de mélange. Malgré ceci, le reste des valeurs sont assez proches des mesures. La validation est accomplie sauf dans la zone de mélange. 


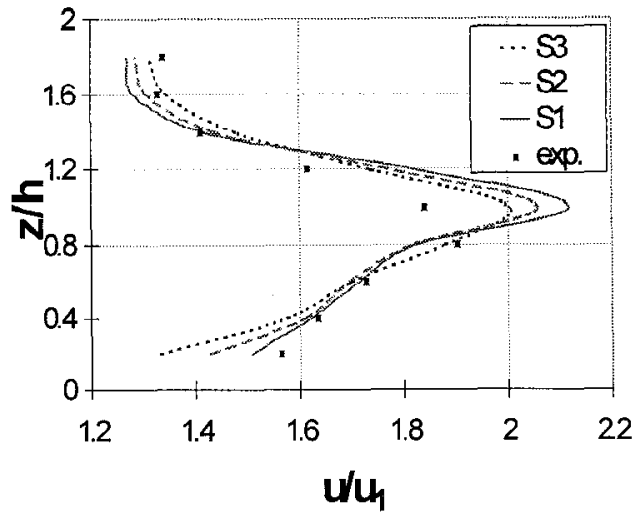

Figure 14 Profils de vitesse $x=4 m$ pour le cas de stratification stable avec le modèle k- $\varepsilon$. Temperature profiles at $x=4 m$

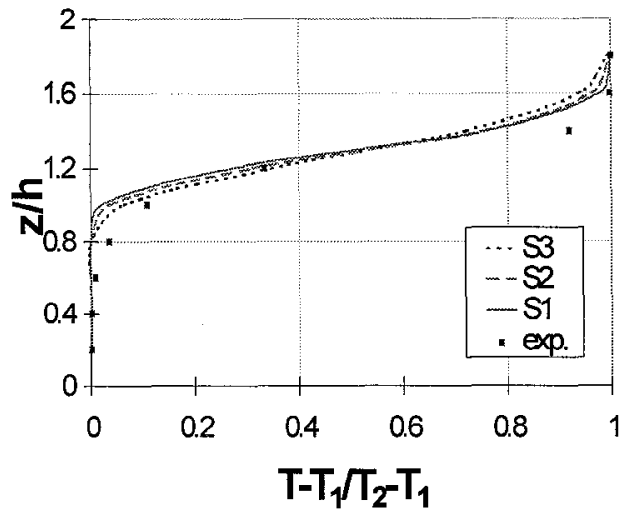

Figure 16 Profils de température $x=4 \mathrm{~m}$ pour le cas de stratification stable avec le modèle k- $\varepsilon$. Temperature profiles at $x=4 m$

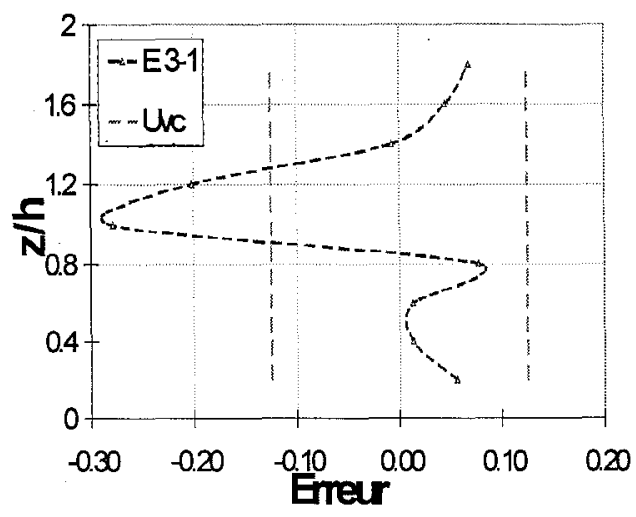

Figure 15 Validation de la vitesse pour le cas de stratification stable avec le modèle $k-\varepsilon$. Velocity convergence validation for study 3-1

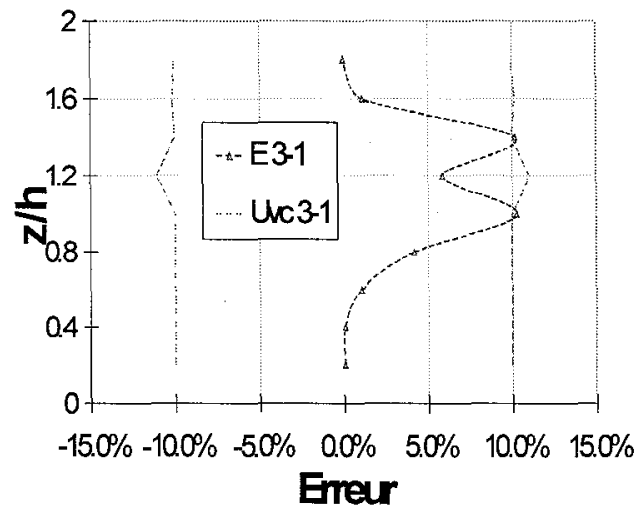

Figure 17 Validation de la température pour le cas de stratification stable avec le modèle $\mathrm{k}-\varepsilon$. Velocity convergence validation for study 3-1

En ce qui concerne la température (voir Figure 16), deux points près de la zone de mélange se trouvent légèrement écartés par rapport aux donnés expérimentaux, raison pour laquelle on aperçoit deux points d'erreur au limite de la zone de validation, Figure 17. Cependant d'une façon globale, les étapes de vérification et validation ont été accomplies.

\section{Conclusions}

On présente dans cet article la modélisation numérique d'un écoulement turbulent dans un canal composé de deux couches ayant des vitesses et des températures 
différentes, définissant des cas de stratification stable ou instable. Cette étude a été réalisée expérimentalement par Viollet (1980). La vérification et validation du code de simulation TELEMAC 3D (Janin et al., 1992) avec la méthode de Colleman et Stern (1997) a été accomplie dans les deux études pour la vitesse et la température pour les cas de stratification stable et instable. Le rayon de convergence numérique a atteint une valeur inférieur à 1 , ce qui représente une convergence monotone; avec possibilité de correction. Le code TELEMAC 3D a été accrédité pour la modélisation de ce cas. Les modèles de turbulence de longueur de mélange et $\mathrm{k}-\varepsilon$ ont été vérifiés et validés.

\section{Références}

* Colleman W.H., Stern F.,(1997), «Uncertainties in CFD Code Validation, ASME J. Fluids Eng.

* Janin J.M., Lepeintre F., Péchon P.,(1992), «Telemac-3D : A finite element code to solve 3D free surface flow problems ", Computer modelling of seas and coastal regions, Southampton, (UK)

* Salaün P., (1980), «Etude expérimental de mélange dans un écoulement stratifié instable », Rapport de stage, LNH-DER-EDF E44.80-23, France, pp 25-27

* Viollet P.L.,(1980), «Turbulent mixing in a two layers stratified flow», 2nd international symposium on stratified flows, Trondheim, Norvege, pp 315-325 